
\title{
R Reserach S Suare \\ Comparison Of Teriparatide And Zoledronic Acid In Post-Menopausal Women With Multiple Osteoporotic Vertebral Fractures
}

\section{Yichao Ji}

The First Affiliated Hospital of Soochow University

Linlin Zhang ( $\square$ zhangdoublelin@139.com )

The First Affiliated Hospital of Soochow University

\section{Huilin Yang}

The First Affiliated Hospital of Soochow University

\section{Research Article}

Keywords: Teriparatide, Zoledronic Acid, multiple osteoporotic vertebral fractures, Bone mineral density, Serum bone metabolism markers

Posted Date: May 6th, 2021

DOl: https://doi.org/10.21203/rs.3.rs-476996/v1

License: (c) (i) This work is licensed under a Creative Commons Attribution 4.0 International License. Read Full License 


\section{Abstract}

Objective: This retrospective comparative study was aimed to make a comparison between Teriparatide and Zoledronic Acid to provide evidence for the sequential treatment of multiple vertebral fractures caused by osteoporosis of postmenopausal women.

Methods: A sum of 106 post-menopausal women undergoing non-operative treatment for recent multiple osteoporotic vertebral fractures between April,2015 and June,2019 were reviewed retrospectively. 44 patients had a treatment of subcutaneous injection of 20 micrograms teriparatide once a day (TPTD group), while 62 patients received $5 \mathrm{mg}$ of zoledronic acid given by an intravenous (IV) infusion once a year (ZOL group). The assessment methods included the general information of patients, the number of new fractures and changes in bone turnover markers, bone mineral density(BMD), back pain and healthrelated quality of life.

Results: Teriparatide was more effective than zoledronic acid in reducing the risk of new vertebral fractures. The lumbar spine BMD rosed significantly in either treatment groups relative to the baseline (P区 0.05). However, the TPTD group increased more than the ZOL group. The hip joint BMD also increased significantly in all groups between months 0 and 12 relative to the baseline $\mathbb{P} \varangle 0.05 \rrbracket$, no significant differences were found between the changes of two groups after 6 months, but the TPTD group was significantly higher than the ZOL group at 12 months $\triangle P \otimes 0.05)$. In the TPTD group, the values of TP1NP increased $55.9 \%$ and $70.8 \%$ from baseline $(71.69 \pm 33.45 \mathrm{ng} / \mathrm{ml} \mathrm{P} \otimes 0.05)$. The values of $\beta$-CTX increased from baseline $(0.807 \pm 0.657 \mathrm{ng} / \mathrm{ml})$ to $(0.842 \pm 0.528) \mathrm{ng} / \mathrm{ml}$ at 6 months and the value got back to the baseline at 12 months after the TPTD used. In the ZOL group, the values of TP1NP and $\beta$-CTX decreased significantly compared with baseline $(69.55 \pm 39.15 \mathrm{ng} / \mathrm{ml}, 0.691 \pm 0.456 \mathrm{ng} / \mathrm{ml}) 6$ months after treatment,

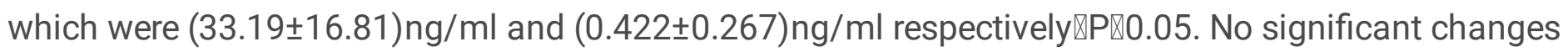
were found 12 months after treatment compared with the conditions of 6 months(Pख0.05). In both TPTD and ZOL treatment groups, there were significant and clinically relevant improvements in back pain as well as health-related quality of life indicators compared with baseline.

Conclusions: TPTD is more advantageous in reducing new fractures, increasing lumbar spine and hip joint BMD and reducing patients' back pain compared with ZOL. TPTD significantly increased marker of bone formation along with slightly increased marker of bone resorption, while ZOL decreased them. This provides a theoretical basis for the apply of Zoledronic acid after discontinuation of teriparatide.

\section{Introduction}

Osteoporosis is a chronic disease of unknown etiology and the characteristics of osteoporosis are microarchitectural deterioration and skeletal fragility. With the rapid speed of our country's aging process, osteoporosis has become a common disease that can not be ignored. According to rough statistics, there are more than 90 million patients with osteoporosis in China. The brittle fracture caused by osteoporosis not only seriously affects the quality of life and life expectancy of the elderly, but also brings a huge 
burden to the society and families. Postmenopausal osteoporosis usually occurs 5-10 years after menopause. the main reason is the decrease of bone mass and the change of bone tissue structure caused by the lack of estrogen, which increases the brittleness of bone and is easy to fracture.

Osteoporotic fractures are most common in the spine (the most common in the thoracolumbar segment). Osteoporosis is usually a silent disease that precedes the fracture until the sentinel fracture event is discovered. The preexisting vertebral fracture is an significant hazard factor for the subsequent vertebral fracture[ $\left.{ }^{1}\right]$, which means patients who have had recent osteoporotic fracture may have a second fracture soon, and finally develop into multiple osteoporotic vertebral fractures if without any intervening measure. Therefore, anti-osteoporosis therapy is of great significance to improve multiple osteoporotic vertebral fractures.

At present, there are mainly three kinds of clinical drugs for osteoporosis: basic supplement (calcium and vitamin D), anti-bone resorption drugs and osteogenic drugs. The Anti-bone resorption drugs target osteoclast function and osteoclast formation, including bisphosphonates (BPs), selective estrogen receptor modulators (SERMs), hormone replacement therapy (HRT) and denosumab (Dmab). The bone anabolic drugs stimulate the differentiation, function and survival of osteoblasts, which mainly include abaloparatide (ABL), teriparatide (TPTD) and romosozumab (ROMO). Each drug has been shown to significantly improve bone density and decrease the risk of fractures $\left[{ }^{2}\right]$. Teriparatide, a recombinant human 1-34 parathyroid hormone, is the first anabolic drug approved to treat osteoporosis[ $\left.{ }^{3}\right]$. Daily use of teriparatide has an effect of stimulating bone formation, increasing bone mass and reducing the possibility o vertebral and non-vertebral fractures $\left[{ }^{4}\right][5]$. It is shown in previous studies that teriparatide has a role in osteoporosis of post-menopausal female, men and patients taking glucocorticoids for a long time[$\left.{ }^{6}\right]\left[{ }^{7}\right]$. Zoledronic acid (ZOL), a third generation of nitrogenous bisphosphonate, annual intravenous infusion of which $5 \mathrm{mg}$ ensures bisphosphonate available and persistent over 12 months[ $\left.\left.\left.{ }^{8}\right]\right]^{9}\right]$. ZOL is able to rose bone mineral density(BMD) and reduce the risk of osteoporotic fracture through inhibiting the function of osteoclast and inducing apoptosis of osteoclast, ultimately prolonging the overall survival of patients suffer from osteoporosis $\left[{ }^{9}\right]\left[^{10}\right]$.

Both teriparatide and zoledronic acid are effective drugs commonly used in the treatment of osteoporosis in clinical work, which can reduce the incidence of vertebral refracture and increase the bone density of patients. However, few studies have compared the efficacy of the two drugs in postmenopausal women with multiple osteoporotic vertebral fractures.

The retrospective comparative study assessed the number of new fractures, bone mineral density (BMD), $\mathrm{N}$-terminal propeptide of type冈procollagen (TP1NP), beta collagen cross-linked C-terminal peptide ( $\beta$ CTX), Visual Analog Scale (VAS) score for back pain and health-related quality of life (HRQoL) during the observation of patients for 12 months. We hope that this comparison can investigate the efficacy of Teriparatide compared with $\mathrm{ZOL}$ in women of after the menopause with multiple osteoporotic vertebral fracture. By comparing the difference of their efficacy, we can provide the basis for the choice of drugs in 
clinical work. Finally, our aim was to provide a theoretical basis for the sequential treatment of TPTD and ZOL.

\section{Materials And Methods}

\section{Study subjects}

Date of post-menopausal women diagnosed with multiple osteoporotic vetibral fractures at the first affiliated hospital of soochow university between April,2015 and June,2019 were retrieved from the electronic medical records system of this institution. Patients were electable for enrollment if they meet the following criteria: (1) Postmenopausal women aged over 55 years old (2) The patients suffered from multiple osteoporotic vetibral fractures (3) The patients had symptoms of bone pain (4) The quality of life was affected by the osteoporosis (5) The patients had a bone mineral density T score of -2.5 or less testing by dual-energy $x$-ray absorptiometry (DXA) with multiple fragility-related fractures (6) The patients had a complete relevant treatment data before and after administration of triptapeptide and ZOL. The exclusion criteria was that: (1) Patients with endocrine diseases affecting bone metabolism (pituitary, thyroid, parathyroid, adrenal and gonadal diseases, etc.) (2) Patients with autoimmune diseases (rheumatoid arthritis, systemic lupus erythematosus, thymus diseases, etc.) (3) Patients with kidney and digestive tract diseases that cause vitamin $\mathrm{D}$ and calcium absorption and regulation disorders (4) Patients with long-term use of immunosuppressant, glucocorticoid and other drugs affecting bone metabolism (5) Patients with severe heart, lung, liver, kidney and other organ function impairment (6) Patients had used other antiosteopathic drugs half a year before the study. Finally, a sum of 106 patients were included.

\section{Treatment}

Two groups were divided depending on the medication patients took: 44 patients were treated with teriparatide (TPTD group) while 62 received Zoledronic Acid (ZOL group). Baseline characteristics and the number of existing fractures are listed in Table 1. BMD, TP1NP, $\beta$-CTX, VAS score for back pain and EQ-5D (EuroQol questionnaire with 5 dimensions and 5 levels) for HRQoL were measured in all subjects before treatment. The TPTD group had a treatment of subcutaneous injection of 20 micrograms teriparatide once a day. In the ZOL group, $5 \mathrm{mg}$ of zoledronic acid was given by an intravenous (IV) infusion once a year. Both groups received oral calcium and vitamin $D$ supplements. The observation time was 12 months. BMD, TP1NP and $\beta$-CTX were reviewed 6 and 12 months after treatment, the VAS score and HRQoL were re-evaluated at the same time.

\section{Date collection}

General information of patients (age, height, weight, BMI) was counted. The number of vertebral fractures that had occurred at the beginning of treatment was counted as well.

Efficacy of teriparatide and zoledronic acid treatment were assessed by the number of new fractures and changes in BMD, TP1NP, $\beta$-CTX, VAS score for back pain and EQ-5D for HRQoL. 


\section{New fractures}

The number of clinical new fractures were counted every 6 months. The occurrence of a clinical vertebral fractures were defined as new vertebral fractures discovered at follow-up after medication and later confirmed on radiograph.

\section{Bone mineral density}

The BMD was measured by dual energy X-ray absorptiometry (DXA). The BMD of lumbar spine (L2-4) and all hip joint were assessed at baseline and 6,12 months after treatment.

\section{Bone turnover markers}

Bone turnover markers include beta collagen cross-linked C-terminal peptide ( $\beta$-CTX) and $N$ terminal propeptide of type冈procollagen (TP1NP) of the patients before treatment and after treatment for 6 months, 12 months were measured by venous blood taken from patients on an empty stomach. The serum samples were obtained by centrifugation, the markers of bone turnover were detected by ELISA test after separating.

\section{Back pain}

Back pain was assessed by patients themself through an 11-point digital back pain rating scale, with 0 indicating no back pain and 10 indicating most severe back pain.

\section{Health-related quality of life}

HRQoL was assessed using the EQ-5D. The EQ-5D consist of self-care, usual activities, mobility, pain/discomfort and anxiety/depression dimensions, which were self-assessed by patients with a 5grade scale of none, slight, moderate, severe or extreme. The scores were ranged from 0 (worst) to 100 (best) to represent overall health status.

\section{Statistical analysis}

The statistical analysis was made using SPSS 20.0 statistical software(IBM, USA). The measurement data of normal distribution were expressed as mean \pm standard deviation, the differences between groups were tested by $t$ test of independent samples. $P<0.05$ was considered statistically significant.

\section{Results}

\section{Baseline demographics and clinical characteristics}

There were 44 patients included in the TPTD group, while 62 patients in the ZOL group. Baseline demographics and clinical characteristics were similar between the two groups (Table 1). In the TPTD group ,the average age of the 44 eligible patients was $(67.8 \pm 7.1)$ years old, the mean BMI was $(23.9 \pm$ $2.5) \mathrm{kg} / \mathrm{m}^{2}$, the mean height was $(157.6 \pm 6.9) \mathrm{cm}$ and the mean weight was $(60.8 \pm 6.6) \mathrm{kg}$. In the ZOL 
group, the average age was $(69.1 \pm 8.4)$ years old, the mean BMI was $(23.4 \pm 3.2) \mathrm{kg} / \mathrm{m}^{2}$, the mean height was $(158.3 \pm 7.5) \mathrm{cm}$ and the mean weight was $(61.1 \pm 6.9) \mathrm{kg}$. The number of fractures before treatment was similar in both groups(Table 1 ).

Table 1

Baseline characteristics and previous vertebral fractures

\begin{tabular}{|llll|}
\hline & TPTD group $(n=44)$ & ZOL group $(n=62)$ & P-value \\
\hline Age & $67.8 \pm 7.1$ & $69.1 \pm 8.4$ & 0.735 \\
\hline Mean height & $157.6 \pm 6.9$ & $158.3 \pm 7.5$ & 0.449 \\
\hline Mean weight & $60.8 \pm 6.6$ & $61.1 \pm 6.9$ & 0.746 \\
\hline Mean BMI & $23.9 \pm 2.5$ & $23.4 \pm 3.2$ & 0.057 \\
\hline Vertebral fractures & & & \\
\hline 2 & $25(57 \%)$ & $36(58 \%)$ & \\
\hline 3 & $12(27 \%)$ & $16(26 \%)$ & \\
\hline$\geq 4$ & $7(16 \%)$ & $10(16 \%)$ & \\
\hline
\end{tabular}

\section{New fractures}

In the TPTD group, there were 2 patients developed new vertebral fractures after treatment with teriparatide. In the ZOL group, there were 5 patient with new vertebral fracture after treatment.

Teriparatide was associated with a greater reduction in the risk of new vertebral fractures than zoledronic acid (odds ratio $=0.54 ; 95 \%$ confidence interval, $0.10-2.93$ ). Compared with zoledronic acid, treatment with teriparatide resulted in a significantly greater reduction in fracture risk and the relative risk reduction was $43.64 \%(P<0.05)$.

\section{Bone mineral density}

The lumbar spine BMD at baseline was $(0.705 \pm 0.07) \mathrm{g} / \mathrm{cm}^{2}$ in the TPTD group and $(0.703 \pm 0.05) \mathrm{g} / \mathrm{cm}^{2}$ in the ZOL group. There were no significant differences between the groups $(P>0.05)$. The lumbar spine BMD of patients after the treatment of teriparatide for 6 and 12 months were $(0.772 \pm 0.06) \mathrm{g} / \mathrm{cm}^{2}$ and $(0.815 \pm 0.06) \mathrm{g} / \mathrm{cm}^{2}$. About the group of ZOL, the lumbar spine BMD of patients 6 and 12 months after treatment were $(0.723 \pm 0.05) \mathrm{g} / \mathrm{cm}^{2}$ and $(0.736 \pm 0.06) \mathrm{g} / \mathrm{cm}^{2}$. The lumbar spine BMD were significantly higher in both groups compared to the baseline $(P<0.01$ for all within-group comparisons). However, the TPTD group increased more than the ZOL group.

The left and right hip joint BMD also increased significantly in all groups between months 0 and 12 relative to the baseline( $\mathrm{P}<0.05$ for all within group comparisons). No significant differences were found between the changes of two groups after 6 months, but the TPTD group was significantly higher than the ZOL group at 12 months(Table 2). 
Table 2

Changes in bone density at various sites

\begin{tabular}{|c|c|c|c|c|}
\hline & & TPTD group & ZOL group & P-value \\
\hline \multirow[t]{3}{*}{ lumbar spine } & Baseline & $0.705 \pm 0.07$ & $0.703 \pm 0.05$ & 0.917 \\
\hline & After 6 months & $0.772 \pm 0.06$ & $0.723 \pm 0.05$ & $\varangle 0.01$ \\
\hline & After 12 months & $0.815 \pm 0.06$ & $0.736 \pm 0.06$ & $\varangle 0.01$ \\
\hline \multirow[t]{3}{*}{ Left hip joint } & Baseline & $0.670 \pm 0.11$ & $0.689 \pm 0.11$ & 0.371 \\
\hline & After 6 months & $0.699 \pm 0.11$ & $0.696 \pm 0.11$ & 0.908 \\
\hline & After 12 months & $0.754 \pm 0.10$ & $0.704 \pm 0.11$ & 0.02 \\
\hline \multirow[t]{3}{*}{ Right hip joint } & Baseline & $0.681 \pm 0.12$ & $0.700 \pm 0.10$ & 0.353 \\
\hline & After 6 months & $0.707 \pm 0.11$ & $0.706 \pm 0.10$ & 0.963 \\
\hline & After 12 months & $0.761 \pm 0.12$ & $0.716 \pm 0.10$ & 0.02 \\
\hline
\end{tabular}

\section{Serum bone metabolism markers}

In the TPTD group, the values of TP1NP rose after the beginning of TPTD treatment, which were (111.76 $\pm 39.13) \mathrm{ng} / \mathrm{ml}$ and $(122.42 \pm 50.72) \mathrm{ng} / \mathrm{ml}$ at 6 and 12 months, respectively, increasing $55.9 \%$ and $70.8 \%$ from baseline $(71.69 \pm 33.45 \mathrm{ng} / \mathrm{ml}, P<0.05)$. At the same time, the values of $\beta$-CTX increased from baseline $(0.807 \pm 0.657 \mathrm{ng} / \mathrm{ml})$ to $(0.842 \pm 0.528) \mathrm{ng} / \mathrm{ml}$ after 6 months and recovered to baseline after 12 months use of the TPTD. In the ZOL group, the values of TP1NP and $\beta$-CTX decreased significantly from baseline $(69.55 \pm 39.15 \mathrm{ng} / \mathrm{ml}, 0.691 \pm 0.456 \mathrm{ng} / \mathrm{ml}) 6$ months after treatment, were $(33.19 \pm 16.81)$ and $(0.422 \pm 0.267) \mathrm{ng} / \mathrm{ml}$ respectively $(P<0.05)$. No significant changes were found 12 months after treatment compared with the conditions of 6 months $(P>0.05)$. (Table 3$)$

Table 3

Changes of $\beta$-CTX and TP1NP

\begin{tabular}{|c|c|c|c|c|}
\hline & & TPTD group & ZOL group & P-value \\
\hline \multirow[t]{3}{*}{ TP1NP } & Baseline & $71.69 \pm 33.45$ & $69.55 \pm 39.15$ & 0.859 \\
\hline & After 6 months & $111.76 \pm 39.13$ & $33.19 \pm 16.81$ & $<0.01$ \\
\hline & After 12 months & $122.42 \pm 50.72$ & $26.42 \pm 13.76$ & $<0.01$ \\
\hline \multirow[t]{3}{*}{$\beta-C T X$} & Baseline & $0.807 \pm 0.657$ & $0.691 \pm 0.456$ & 0.404 \\
\hline & After 6 months & $0.842 \pm 0.528$ & $0.422 \pm 0.267$ & $<0.01$ \\
\hline & After 12 months & $0.764 \pm 0.541$ & $0.375 \pm 0.223$ & $<0.01$ \\
\hline
\end{tabular}


There were significant and clinically relevant improvements in back pain and health-related quality of life indicators compared with baseline in both TPTD and ZOL group. At baseline, back pain was similar in both two groups. The changes from baseline were significant in each treatment group $(p<0.05$ in each group, Fig. 1). The treatment of TPTD was superior to that of $Z O L$ in reducing back pain $(P<0.05)$. The EQ VAS recorded the self-reported health of respondents on a visual scale of 0-100, with 0 being the worst health you can imagine and 100 being the best health you can imagine. The mean EQ VAS of the TPTD group and the ZOL group were $64.5 \pm 21.2 \mathrm{~mm}$ and $65.1 \pm 20.2 \mathrm{~mm}$ at baseline, respectively. There was no statistically significant difference between treatment groups at each time point $(P>0.05)$, but statistical significance was found within each treatment group compared with baseline $(p<0.05$ in each group, Fig. 2).

\section{Discussion}

Both teriparatide and Zoledronic Acid are commonly used drugs in clinical practice, they have their own advantages and disadvantages. Teriparatide plays an osteogenic role by directly stimulating osteoblasts at early stage, preventing the apoptosis of osteoblasts and increasing the activity of osteoblasts $\left[{ }^{11}\right]\left[{ }^{12}\right]$ $\left[{ }^{13}\right]$. In various animal models, teriparatide has been shown to be able to accelerate fracture healing $\left[{ }^{14}\right]\left[{ }^{15}\right]$ $\left.{ }^{16}\right]$. Zoledronic acid, administering intravenously of which once a year at a dose of $5 \mathrm{mg}$ was approved for the treatment of post-menopausal, male and glucocorticoid-induced osteoporosis[10]. Zoledronic Acid has a strong inhibitory effect on bone absorption, which can effectively improve the filling of the remodeled space by secondary mineralization and significantly reduce vertebral/non-vertebral fractures $\left[{ }^{17}\right]$.

In this study, the clinical efficacy of TPTD and ZOL in the treatment of multiple osteoporotic vertebral fractures in post-menopausal women was observed by a retrospective comparative study. Patients with multiple osteoporotic vertebral fractures treated with TPTD and ZOL were selected to make statistics on the number of new fractures after each therapy, along with the changes in BMD, bone metabolism markers, back pain and HRQoL before and after 6 and 12 months of treatment. A comparison was made after date collecting.

In the course of the survey, we found that many patients did not use the teriparatide for 12 months possibly because they were scheduled to use it for less than 12 months or the lack of significant effects in their mind, adverse reactions and high drug prices. Since the reasons above, we think that if the medicine can be further promoted, more people will know the clear efficacy, at the same time improve the process, reduce side effects and lower drug prices, It is believed that more patients will choose to complete a one-year course of treatment. In contrast, ZOL is relatively inexpensive and only needs to be given by an intravenous (IV) infusion once a year, making it convenient for elderly patients, which most patients can accept.

In addition, only a few of the patients with teriparatide had dizziness, nausea, vomiting, limb pain, blurred vision and other adverse reactions. Most of the symptoms were mild, which was consistent with the 
results of previous studies $\left[{ }^{18}\right]\left[{ }^{19}\right]$. In patients receiving ZOL by an intravenous (IV) infusion , the side effects were no more than TPTD.

The results of this study showed that post-menopausal women subcutaneously injected with teriparatide at $20 \mathrm{ug} / \mathrm{d}$ and intravenous infusion of $5 \mathrm{mg}$ ZOL once a year, whose lumbar spine BMD were improved compared with baseline. After 12 months of treatment, there were more significant improvement in the lumbar spine BMD. The TPTD group increased more than the ZOL group. This study also found that the BMD of left and right hip joint increased significantly after 12 months of both medications. There were no significant differences between the two groups after 6 months, however, differences were found after 12 months. The reason may be that the TPTD stimulates new bone formation and produces a mineralized bone matrix, which is relatively heterogeneous and has a stable average mineral content, while the ZOL slows down bone conversion, prolongs secondary mineralization and a uniform and highly mineralized bone matrix is gradually formed $\left.{ }^{20}\right]$. Although both the two drugs increase the clinical measurement of $B M D$, the underlying mechanism for increased bone density is quite different.

In clinical practice, the combined measurement of bone formation markers and bone resorption markers is often used to predict the bone loss rate, so as to evaluate the therapeutic effect of osteoporosis $\left[{ }^{21}\right]$. TP1NP is a specific indicator of osteoblast function, bone conversion and osteogenesis, which is produced and secreted by osteoblasts. TPINP is the most sensitive indicator of bone formation at present $\left.{ }^{22}\right]$. $\beta$-CTX is a metabolite of collagen in bone tissue, which mainly reflects the absorption of bone $\left.{ }^{23}\right]$. After 6 months of TPTD administration, TP1NP was significantly increased. The increase was more pronounced at 12 months. At 6 months of administration, there was a slight increase in $\beta$-CTX, but not statistically significant. This may be related to the occurrence of fractures in patients prior to administration and the possible elevation of $\beta$-CTX shortly after the fracture, or to the slight bone resorption effects of Teriparatide during short-term use. After 12 months of administration, $\beta$-CTX begins to decline and gradually returns to baseline levels. In conclusion, bone formation increased rapidly after teriparatide treatment, but osteoblasts subsequently stimulated osteoclasts which lead to the increasing of bone resorption. In contrast, in the ZOL group, TP1NP and B-CTX decreased significantly at 6 months and remained at the same level at 12 months, slightly increasing compared with 6 months. This indicates that ZOL has more advantages in reducing bone resorption.

The effects of the bone formation therapy are reversible. Several clinical trials had shown a decrease in BMD after discontinuation of teriparatide $\left[{ }^{24}\right]$. According to the results of this study, we believe that the use of zoledronic acid after discontinuation of teriparatide can be considered to reduce the increase in bone absorption caused by teriparatide, further improve the bone density during discontinuation and maintain the anti-fracture effect of teriparatide.

Patients in the TPTD and ZOL groups reported similar results, with improvements in both back pain and HRQoL. These improvements are consistent with the results of a double-blind, actively controlled study of patients with vertebral fractures and back pain[25]. TPTD acts on nerve cells and bone cells, and genes associated with neuroprotection and inflammation are involved in this process[26]. ZOL plays an 
analgesic role mainly by inhibiting osteoclasts and inflammation[27][28][29]. The difference in the effect of improving back pain may be due to the different mechanism of action.

Several limitations of current research should be noted. On account of the nature of retrospective investigation, BMD of various parts and serum bone markers can only be collected from a very limited number of patients. Patients were not randomly assigned to different treatment groups. In addition, we did not examine patients' incomes; However, because Teriparatide is not covered by health insurance, patients who receive Teriparatide treatment are generally wealthier than those who do not. In our study, this may have led to patient selection bias. Patients were followed up for only 12 months after treatment, and we did not know the efficacy after 12 months. Baseline characteristics were similar between the two groups before treatments, differences were found only after patients received different therapeutic interventions. To further assess the efficacy of Teriparatide and Zoledronic Acid in women with postmenopausal osteoporosis, it is necessary to conduct more randomized controlled trials with longer follow-up periods and larger sample sizes.

\section{Conclusions}

At present, anti-bone resorption preparation is the most commonly used drug for osteoporosis treatment in China, among which Zoledronic Acid plays a role mainly in reducing osteoclast activity and bone transformation. Teriparatide has a dual effect on bone metabolism. Small doses of intermittent administration can promote the formation of bone on the one hand and relatively slow down the rate of bone absorption on the other hand, so as to increase bone density through the increase of endogenous bone[30]. Based on the above information, we can conclude that both TPTD and ZOL have good effects in the treatment of post-menopausal multiple osteoporotic vertebral fractures. TPTD is more advantageous in reducing new fractures, increasing lumbar spine and hip joint BMD and reducing patients' back pain compared with ZOL. TPTD significantly increased marker of bone formation and slightly increased bone resorption markers, while ZOL decreased them. This provides a theoretical basis for the use of zoledronic acid after discontinuation of teriparatide.

\section{Declarations}

Ethical approval and consent to participate: This research study was conducted retrospectively from data obtained for clinical purposes and was in accordance with the ethical standards of the institutional and national research committee and with the 1964 Helsinki Declaration. The Human Investigation Committee (IRB) of Soochow University approved this study.Informed consent was obtained from all individual participants included in the study.

Consent for publication: The participant has consented to the publication.

Availability of data and materials: The datasets used or analysed during the current study are available from the corresponding author on reasonable request. 
Funding: No funding was received to assist with the preparation of this manuscript.

Competing interests: Yichao Ji, Linlin Zhang, Huilin Yang declare that they have no conflict of interest.

Authors' contributions: Huilin Yang contributed to the study conception and design. Data collection and analysis were performed by Yichao Ji. The first draft of the manuscript was written by Yichao Ji. Linlin Zhang revising the manuscript critically for important intellectual contentand. All authors commented on previous versions of the manuscript. All authors read and approved the final manuscript.

Acknowledgements: The authors would like to thank the staffs at the First Affiliated Hospital of Soochow University for their assistance throughout the study.

All data generated or analysed during this study are included in this published article and its supplementary information files.

\section{References}

[1] Eisman, J. A. , Bogoch, E. R. , Dell, R. , Harrington, J. T. , Mckinney, R. E. , \& Mclellan, A. , et al. (2012). Making the first fracture the last fracture: asbmr task force report on secondary fracture prevention. Journal of Bone \& Mineral Research, 27(10), 2039-2046.

[2] Black, D. M. , \& Rosen, C. J. . (2016). Clinical practice. Postmenopausal osteoporosis.

[3] Zebaze, R. M. , Libanati, C. , Austin, M. , Ghasem-Zadeh, A. , \& Seeman, E. . (2013). Differing effects of denosumab and alendronate on cortical and trabecular bone. Bone, 59(2), 173-179.

[4] Oswald, A. J. , Berg, J. , Milne, G. , \& Ralston, S. H. . (2014). Teriparatide treatment of severe osteoporosis reduces the risk of vertebral fractures compared with standard care in routine clinical practice. Calcified Tissue International, 94(2), 176.

[5] Walsh, J. B. , Lems, W. F. , Karras, D. , Langdahl, B. L. , Ljunggren, O. , \& Fahrleitner-Pammer, A. , et al. (2012). Effectiveness of teriparatide in women over 75 years of age with severe osteoporosis: 36-month results from the european forsteo observational study (efos). Calcif Tissue Int, 90(5), 373-383.

[6] Adler, \& Robert, A. . (2018). Update on Osteoporosis in Men.

[7] Tanaka, I. , Tanaka, Y. , Soen, S. , \& Oshima, H. . (2020). Efficacy of once-weekly teriparatide in patients with glucocorticoid-induced osteoporosis: the tower-go study. Journal of Bone and Mineral Metabolism, 1-10.

[8] Huang, S. , Lin, H. , Zhu, X. , Chen, X. , Fan, L. , \& Liu, C. . (2014). Zoledronic acid increases bone mineral density and improves health-related quality of life over two years of treatment in chinese women with postmenopausal osteoporosis. Endokrynologia Polska, 65(2), 96-104. 
[9] Maricic, M. . (2010). The role of zoledronic acid in the management of osteoporosis. Clinical Rheumatology, 29(10), 1079-1084.

[10] Dhillon, S. . (2016). Zoledronic acid (reclast ${ }^{\circledR}$, aclasta $\left.{ }^{\circledR}\right)$ : a review in osteoporosis. Drugs.

[11] John, M. T. , Sims, N. A. , \& Ego, S. . (2021). Physiological and pharmacological roles of pth and pthrp in bone using their shared receptor, pth1r. Endocrine Reviews.

[12] Kim SW, Pajevic PD, Selig M, Barry KJ, Y ang JY , Shin CS, Baek WY , Kim JE, Kronenberg HM. . (2012). Intermittent parathyroid hormone administration converts quiescent lining cells to active osteoblasts. Journal of Bone and Mineral Research, 27(10).

[13] Bellido T, Ali AA, Plotkin LI, Fu Q, Gubrij I, Roberson PK, Weinstein RS, O'Brien CA, Manolagas SC, Jilka RL. .(2003). Proteasomal degradation of runx2 shortens parathyroid hormone-induced anti-apoptotic signaling in osteoblasts. a putative explanation for why intermittent administration is needed for bone anabolism. Journal of Biological Chemistry, 278(50), 50259-72.

[14] Zhang, W. , Zhu, J. , Ma, T. , Liu, C. , Hai, B. , \& Du, G. , et al. (2017). Comparison of the effects of onceweekly and once-daily rhpth (1-34) injections on promoting fracture healing in rodents. Journal of Orthopaedic Research.

[15] Rowshan, H. H. , Parham, M. A. , Baur, D. A. , Mcentee, R. , Cauley, E. , \& Carriere, D. T. , et al. (2010). Effect of intermittent systemic administration of recombinant parathyroid hormone (1-34) on mandibular fracture healing in rats. J Oral Maxillofac Surg, 68(2), 260-267.

[16] A, M. K. , A, E. K. S. , B, C. W. , C, M. W. , A, L. K. , \& A, S. S. , et al. (2010). Effect of human parathyroid hormone hpth (1-34) applied at different regimes on fracture healing and muscle in ovariectomized and healthy rats. Bone, $47(3), 480-492$.

[17] Seeman, E. , \& Martin, T. J. . (2015). Co-administration of antiresorptive and anabolic agents: a missed opportunity. Journal of Bone \& Mineral Research the Official Journal of the American Society for Bone \& Mineral Research, 30(5), 753-764.

[18] Liu, Z. D. , Tao, J. C. , Yi, X. , \& Zhang, C. Y. . (2009). Associations of age-related expression changes of peroxisome proliferator-activated receptor gamma and core binding factor alpha 1 in bone marrow cells with age-related fracture healing impairment in rats. Journal of Clinical Rehabilitative Tissue Engineering Research.

[19]Vandenbroucke, F P, Luyten, Flamaing, \& Gielen. (2017). Pharmacological treatment of osteoporosis in the oldest old. Clinical interventions in aging.

[20] Dempster, D. W. , Roschger, P. , Misof, B. M. , Zhou, H. , Paschalis, E. P. , \& Alam, J. , et al. (2016). Differential effects of teriparatide and zoledronic acid on bone mineralization density distribution at 6 
and 24 months in the shotz study. Journal of Bone \& Mineral Research the Official Journal of the American Society for Bone \& Mineral Research, 1527-1535.

[21] Wei, Y. , Miao, Y. , \& Liu, Z. . (2007). Clinical progress in biochemical markers for bone metabolismreview in biochemical markers for bone metabolism in different countries and regions in premenopausal and postmenopausal women. Chinese Journal of Osteoporosis.

[22] Kyvernitakis, I. , Saeger, U. , Ziller, V. , Bauer, T. , Seker-Pektas, B. , \& Hadji, P. . (2013). The effect of age, sex hormones, and bone turnover markers on calcaneal quantitative ultrasonometry in healthy german men. Journal of Clinical Densitometry, 16(3), 320-328.

[23] Shi, Z. , Jin, H. , Ding, Q. , Ying, J. , \& He, B. . (2019). Bone turnover markers may predict the progression of osteonecrosis of the femoral head in aged males. Annals of Translational Medicine, 7(22), 626-626.

[24] Prince, R. , Sipos, A. , Hossain, A. , Syversen, U. , Ish-Shalom, S. , \& Marcinowska, E. , et al. (2010). Sustained nonvertebral fragility fracture risk reduction after discontinuation of teriparatide treatment. Journal of Bone and Mineral Research, 20(9), 1507-1513.

[25] Hadji, P. , Zanchetta, J. R. , Russo, L. , Recknor, C. P. , Saag, K. G. , \& Mckiernan, F. E. , et al. (2012). The effect of teriparatide compared with risedronate on reduction of back pain in postmenopausal women with osteoporotic vertebral fractures. Osteoporosis International, 23(8), 2141-2150.

[26] Ito, A. , limura, T. , Lee, J. W. , Shimazu, Y. , \& Takao-Kawabata, R. . (2020). Teriparatide relieves ovariectomyinduced hyperalgesia in rats, suggesting the involvement of functional regulation in primary sensory neurons by pth-mediated signaling. Scientific Reports, 10(1), 5346.

[27] A. J. Roelofs, K. Thompson, F. H. Ebetino, M. J. Rogers, F. P. Coxon. (2010). Bisphosphonates: molecular mechanisms of action and effects on bone cells, monocytes and macrophages. Current Pharmaceutical Design, 16(27), - .

[28] Baron, R. , Fe Rrari, S. , \& Russell, R. . (2011). Denosumab and bisphosphonates: different mechanisms of action and effects. Bone, 48(4), 0-692.

[29] Cai, G. , Laslett, L. L. , Aitken, D. , Halliday, A. , Pan, F. , \& Otahal, P. , et al. (2018). Effect of zoledronic acid and denosumab in patients with low back pain and modic change: a proof-of-principle trial. Journal of Bone \& Mineral Research.

[30]Tan Jinmei囚Bao Lihua囚Ma Yuwei囚et al. (2010). The comparison of the clinical outcomes between parathyroid hormone and calcitonin in short-term treatment of postmenopausal osteoporosis. Acta UnivMed Nanjing, 30(5):709-712

\section{Figures}




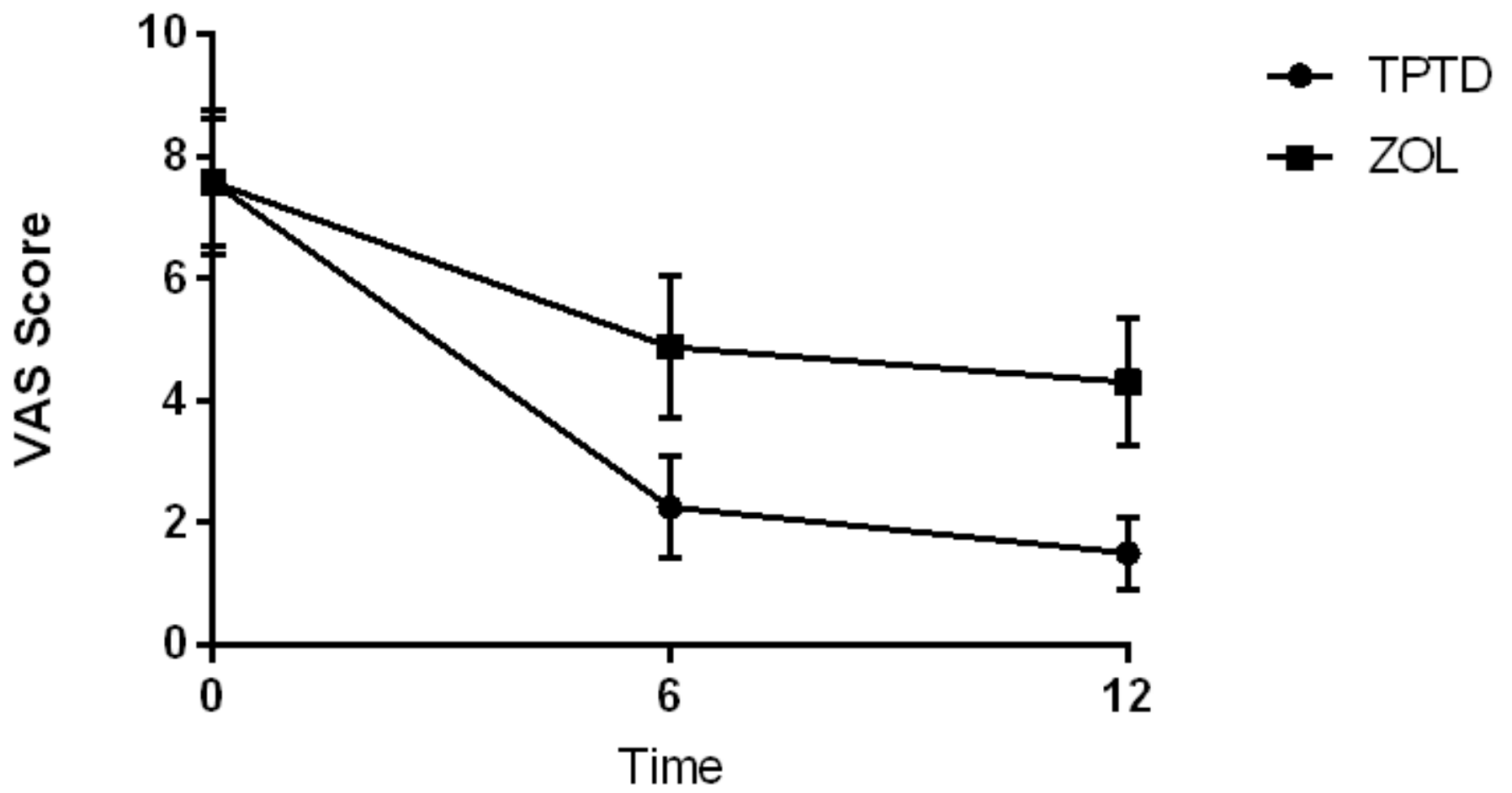

Figure 1

The changes from baseline were significant in each treatment group $(p<0.05$ in each group, figure 1$)$. The treatment of TPTD was superior to that of $Z O L$ in reducing back pain $(P \Downarrow 0.05)$.

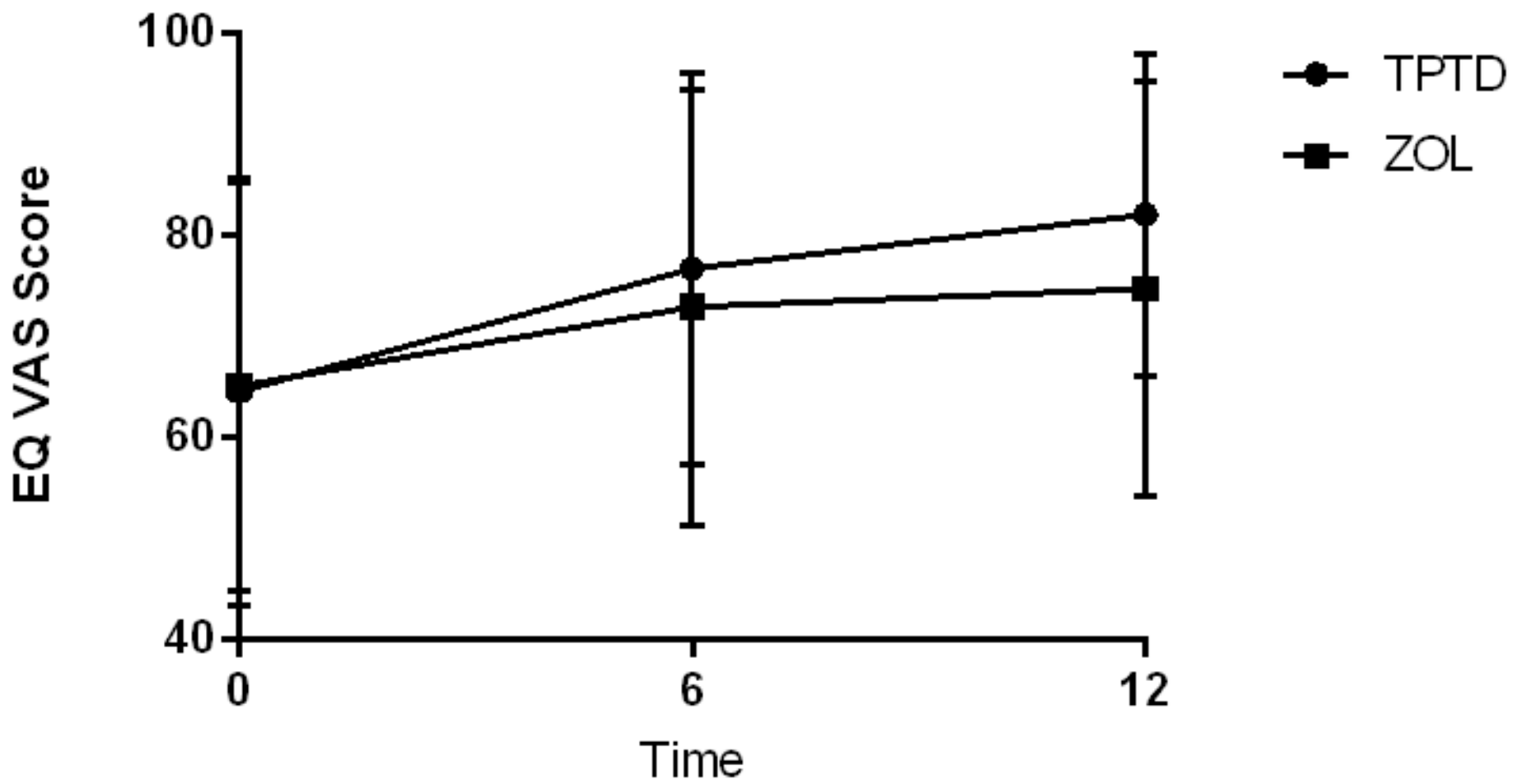


Figure 2

There was no statistically significant difference between treatment groups at each time point $\triangle \mathrm{P} \llbracket 0.05 \rrbracket$, but statistical significance was found within each treatment group compared with baseline $(p<0.05$ in each group)

\section{Supplementary Files}

This is a list of supplementary files associated with this preprint. Click to download.

- rawdate.xIsx 